\title{
Behavior of Li abundances in solar-analog stars ${ }^{\star}$
}

\section{Evidence of the connection with rotation and stellar activity}

\author{
Y. Takeda ${ }^{1}$, S. Honda ${ }^{2}$, S. Kawanomoto ${ }^{1}$, H. Ando ${ }^{1}$, and T. Sakurai ${ }^{1}$ \\ 1 National Astronomical Observatory of Japan, 2-21-1 Osawa, Mitaka, Tokyo 181-8588, Japan \\ e-mail: [takeda.yoichi; kawanomoto.satoshi; ando.hys]@nao.ac.jp, sakurai@solar.mtk.nao.ac.jp \\ 2 Gunma Astronomical Observatory, 6860-86 Nakayama, Takayama-Mura, Agatsuma-gun, Gunma 377-0702, Japan \\ e-mail: honda@astron.pref.gunma.jp
}

Received 18 December 2009 / Accepted 2 February 2010

ABSTRACT

\begin{abstract}
Context. We previously attempted to ascertain why the Li I 6708 line-strengths of Sun-like stars differ so significantly despite the superficial similarities of stellar parameters. We carried out a comprehensive analysis of 118 solar analogs and reported that a close connection exists between the $\mathrm{Li}$ abundance $\left(A_{\mathrm{Li}}\right)$ and the line-broadening width $\left(v_{\mathrm{r}+\mathrm{m}}\right.$; mainly contributed by rotational effect), which led us to conclude that stellar rotation may be the primary control of the surface Li content.

Aims. To examine our claim in more detail, we study whether the degree of stellar activity exhibits a similar correlation with the $\mathrm{Li}$ abundance, which is expected because of the widely believed close connection between rotation and activity.

Methods. We measured the residual flux at the line center of the strong Ca II 8542 line, $r_{0}(8542)$, known to be a useful index of stellar activity, for all sample stars using newly acquired spectra in this near-IR region. The projected rotational velocity $\left(v_{\mathrm{e}} \sin i\right)$ was estimated by subtracting the macroturbulence contribution from $v_{\mathrm{r}+\mathrm{m}}$ that we had already established.

Results. A remarkable (positive) correlation was found in the $A_{\mathrm{Li}}$ versus (vs.) $r_{0}(8542)$ diagram as well as in both the $r_{0}(8542)$ vs. $v_{\mathrm{e}} \sin i$ and $A_{\mathrm{Li}}$ vs. $v_{\mathrm{e}} \sin i$ diagrams, as had been expected. With the confirmation of rotation-dependent stellar activity, this clearly shows that the surface $\mathrm{Li}$ abundances of these solar analogs progressively decrease as the rotation rate decreases.

Conclusions. Given this observational evidence, we conclude that the depletion of surface Li in solar-type stars, probably caused by effective envelope mixing, operates more efficiently as stellar rotation decelerates. It may be promising to attribute the low-Li tendency of planet-host $\mathrm{G}$ dwarfs to their different nature in the stellar angular momentum.
\end{abstract}

Key words. stars: abundances - stars: activity - stars: atmospheres - stars: solar-type - stars: rotation

\section{Introduction}

Since Li nuclei are burned and destroyed on their arrival at the hot stellar interior $\left(T \gtrsim 2.5 \times 10^{6} \mathrm{~K}\right)$, we can gain valuable information from the surface Li composition of a star about the past history and the physical mechanism of stellar envelope mixing. It has been known, however, that $\mathrm{Li}$ abundances $\left(A_{\mathrm{Li}}\right)$ in Sun-like stars exhibits puzzling behaviors:

- a markedly large diversity (by more than $\sim 2 \mathrm{dex}$ ) of $A_{\mathrm{Li}}$ is seen despite the similarity of stellar parameters;

- planet-host stars tend to show appreciably lower $A_{\mathrm{Li}}$ than non-planet-host stars (cf. Israelian et al. 2004, 2009).

As these characteristic trends cannot be explained by the naive classical picture of surface Li being determined by age (which relates to the duration time of gradual Li depletion by way of convective mixing) and $T_{\text {eff }}$ (which affects the depth of the convection zone), it has been important to find the hidden parameter(s) responsible for these observed findings.

To elucidate this problem, Takeda et al. (2007, hereinafter referred to as Paper I) conducted an extensive high-precision study of stellar parameters as well as of $A_{\mathrm{Li}}$ for 118 solar analogs and found that $A_{\mathrm{Li}}$ values, exhibiting a large dispersion themselves, are closely correlated with the line-width, which is characterized

^ Based on observations carried out at Okayama Astrophysical Observatory (Okayama, Japan). by the macroscopic velocity dispersion $\left(v_{\mathrm{r}+\mathrm{m}}\right)$ including the rotational as well as the macroturbulent broadening effect.

We then speculated that $v_{\mathrm{e}}$ (equatorial rotation velocity) would be the most important factor affecting $A_{\mathrm{Li}}$, since the starto-star variation in $v_{\mathrm{e}} \sin i$ may be responsible for the spread in $v_{\mathrm{r}+\mathrm{m}}$, any considerable fluctuation in the macroturbulent velocity field among similar solar-type stars being difficult to imagine.

The motivation of the present paper, the second in a series, is to check (or substantiate) the hypothesis that stellar rotation is the decisive factor which determines the surface Li content of solar-analogs. One useful way to accomplish this would be to examine the stellar activity, which is considered to be of dynamo origin and thus deeply related to the intrinsic rotational rate. That is, if we could confirm that $A_{\mathrm{Li}}$ is closely correlated with the degree of activity, our speculation would be reasonably justified.

As an indicator of stellar activity, we adopt $r_{0}(8542) \quad\left(\equiv f_{0} / f_{\text {cont }}\right)$, which is the residual flux (normalized by the continuum) at the line center of Ca II 8542.09, the strongest line of the near-IR 8498/8542/8662 triplet of mulptiplet 2 for the ${ }^{2} \mathrm{D}-{ }^{2} \mathrm{P}^{\mathrm{o}}$ transition. This is known to reflect the chromospheric activity of a star; i.e., as the activity is enhanced, the core flux increases because of the greater amount of filled-in emission from the chromosphere (see, e.g., Linsky et al. 1979). This quantity is known to be well correlated with the more traditional $\mathrm{Ca}$ II $\mathrm{H}+\mathrm{K}$ emission index $\left(\log R_{\mathrm{HK}}^{\prime}\right)$ and thus serves as a useful tool for diagnosing the activity level of 
late-type stars (e.g., Foing et al. 1989; Chmielewski 2000; Busà et al. 2007).

In this study we aim to determine $r_{0}$ (8542) (a measure of stellar activity ${ }^{1}$ ) for each of the 118 stars studied in Paper I (a bona-fide sample of solar analogs), based on our new spectroscopic data obtained at Okayama Astrophysical Observatory, and examine whether or not they show any correlation with $A_{\mathrm{Li}}$, to test our conclusion in Paper I.

The remainder of this paper is organized as follows. In Sect. 2, we describe the observational material and the measurement of $r_{0}(8542)$. Before discussing the results of stellar activity, the projected rotational velocity $\left(v_{\mathrm{e}} \sin i\right)$ for each star is derived in Sect. 3 by appropriately subtracting the contribution of macroturbulence from the macrobroadening parameter $\left(v_{\mathrm{r}+\mathrm{m}}\right)$ discussed in Paper I. The discussion about the resulting relationship between $r_{0}(8542), A_{\mathrm{Li}}$, and $v_{\mathrm{e}} \sin i$ is presented in Sect. 4, where we show that the arguments in Paper I have been confirmed, and our conclusions are summarized in Sect. 5. Two additional appendices are included. Appendix A describes the results of our reanalysis of stellar parameters (including Li abundance) for HIP 41484, since another star (actually HIP 41184) was erroneously observed and analyzed as if it were HIP 41484 in Paper I. Appendix B is devoted to discussing the sensitivity difference between two representative activity indicators, $\mathrm{Ca}$ II triplet in near-IR (multiplet 2) and $\mathrm{Ca}$ II $\mathrm{H}+\mathrm{K}$ lines in violet region (multiplet 1), based on some test results of non-LTE line profiles simulated with trial models.

\section{Residual line-center flux of Ca II 8542}

To acquire data for studying stellar activities from Ca II nearIR triplet, the observations of 118 solar-analog stars (the same sample as in Paper I) were carried out in five different months (2007 February and April; 2008 May, August, and December) by using the HIgh-Dispersion Echelle Spectrograph (HIDES; Izumiura 1999) at the coudé focus of the $188 \mathrm{~cm}$ reflector of Okayama Astrophysical Observatory (OAO). This HIDES, equipped with a $4 \mathrm{~K} \times 2 \mathrm{~K} C \mathrm{CD}$ detector at the camera focus, enabled us to obtain an echellogram covering the wavelength range $\mathrm{e}^{2}$ of 7600-8800 $\AA$ with a resolving power of $R \sim 70000$ (for the normal slit width of $200 \mu \mathrm{m}$ ) in the mode of red crossdisperser. The observational dates for each of the 118 stars are given in Table 1.

The data reduction (bias subtraction, flat-fielding, aperturedetermination, scattered-light subtraction, spectrum extraction, wavelength calibration, continuum normalization) was performed using the "echelle" package of IRAF'. Several spectral

\footnotetext{
${ }^{1}$ Of course, this $r_{0}(8542)$ index depends not only on the chromospheric activity but also on atmospheric parameters such as $T_{\text {eff }}$ (effective temperature), $\log g$ (surface gravity), and $[\mathrm{Fe} / \mathrm{H}]$ (metallicity) (e.g., Mallik 1997; Chmielewski 2000). However, in the present sample of solar analogs similar to each other, the mutual differences of these stellar parameters are of secondary importance and can be neglected to a first approximation.

2 Since the beginning of 2008, three mosaicked CCD chips had become newly available in HIDES, resulting in a three-times wider wavelength coverage than before. Accordingly, for the data of 2008 May, August, and December, spectra in two adjacent wavelength ranges (6300-7600 Åand 8800-10000 ̊) were also recorded in addition to the target region of $7600-8800 \AA$.

3 IRAF is distributed by the National Optical Astronomy Observatories, which is operated by the Association of Universities for Research in Astronomy, Inc., under cooperative agreement with the National Science Foundation.
}

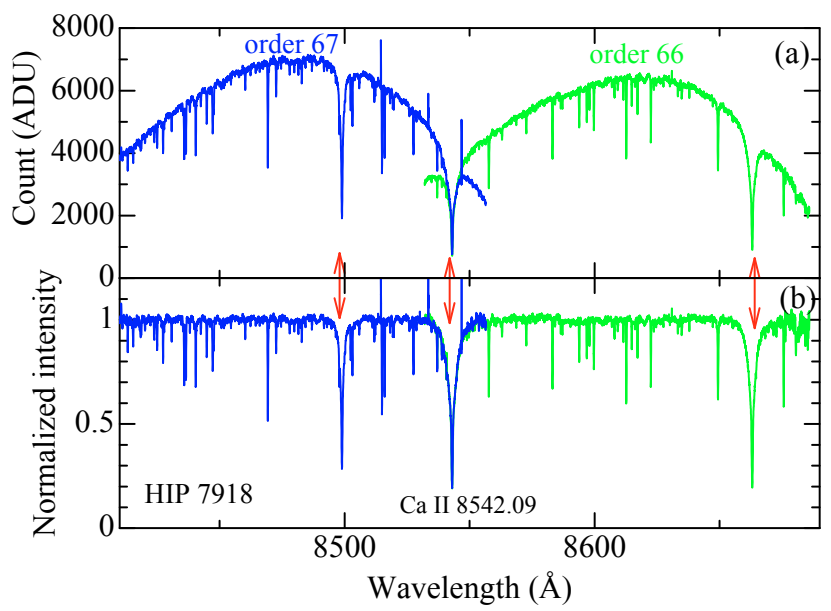

Fig. 1. Example of our HIDES spectrum (for the case of HIP 7918) around the region of Ca II 8498/8542/8662 triplet lines, where spectra of two adjacent orders (66 and 67) are involved. Note that the strongest line (at $8542.09 \AA$ ) among the three, whose central depth was used for estimating the stellar activity, is located near to the edge of each spectrum. a) Unnormalized raw spectrum; b) Normalized spectrum with respect to the continuum level.

frames taken on a night were coadded to improve the signalto-noise ratio, values as high as $S / N \sim 100-200$ being finally accomplished in most cases.

An example spectrum of the Ca II triplet region is shown in Fig. 1 (for HIP 7918). Unfortunately, the strongest Ca II line at $8542.09 \AA$, which we use for diagnosing the stellar activity, is situated close to the edge of the spectral order. Although this did not cause any essential disadvantage for the present purposes, we realized that, because of the difficulty in empirically determining the precise continuum level, a careful readjustment of the continuum normalization was necessary, which we carried out using Kurucz et al.'s (1984) solar spectrum atlas as a reference standard. That is, the wing region $(|\Delta \lambda| \gtrsim 3 \AA$, where $|\Delta \lambda|$ is the distance from the line center of $8542.09 \AA$ ) of each star's spectrum was adjusted so as to match the corresponding wing of the reference solar spectrum. This procedure worked satisfactorily well for all the program stars, as they are analogous to the Sun. These reduced normalized spectra of the core region are displayed in Fig. 2 for all 118 stars (plus Moon), and the superposition of all the core-region spectra is depicted in Fig. 3. The resulting values of the residual flux at the line center of Ca II 8542, $r_{0}\left(\equiv f_{0} / f_{\text {cont }}\right)$, are summarized in Table 1.

\section{Rotational velocity from line width}

To ascertain/determine the effect of rotation on the surface $\mathrm{Li}$ content as well as stellar activity, we need to extract $v_{\mathrm{e}} \sin i$, the projected rotational velocity, from $v_{\mathrm{r}+\mathrm{m}}$ values for the $e$-folding width of the Gaussian macrobroadening function $\left[f(v) \propto \exp \left[-\left(v / v_{\mathrm{r}+\mathrm{m}}\right)^{2}\right]\right.$ including both rotation and macroturbulence, which were already derived in Paper I, by appropriately eliminating the effect of macroturbulence. For this purpose, we adopt the simple line-broadening model also invoked by Takeda \& Tajitsu (2009; cf. Sect. 3.2 therein), where all broadening components are involved in the form of convolution of Gaussians. According to this model, we may assume the relation (see Eq. (1) and footnote 5 of that paper)

$0.94 v_{\mathrm{e}} \sin i \simeq\left(v_{\mathrm{r}+\mathrm{m}}^{2}-v_{\mathrm{mt}}^{2}\right)^{1 / 2}$ 
Y. Takeda et al.: Li abundance, rotation, and stellar activity of solar analogs. II.
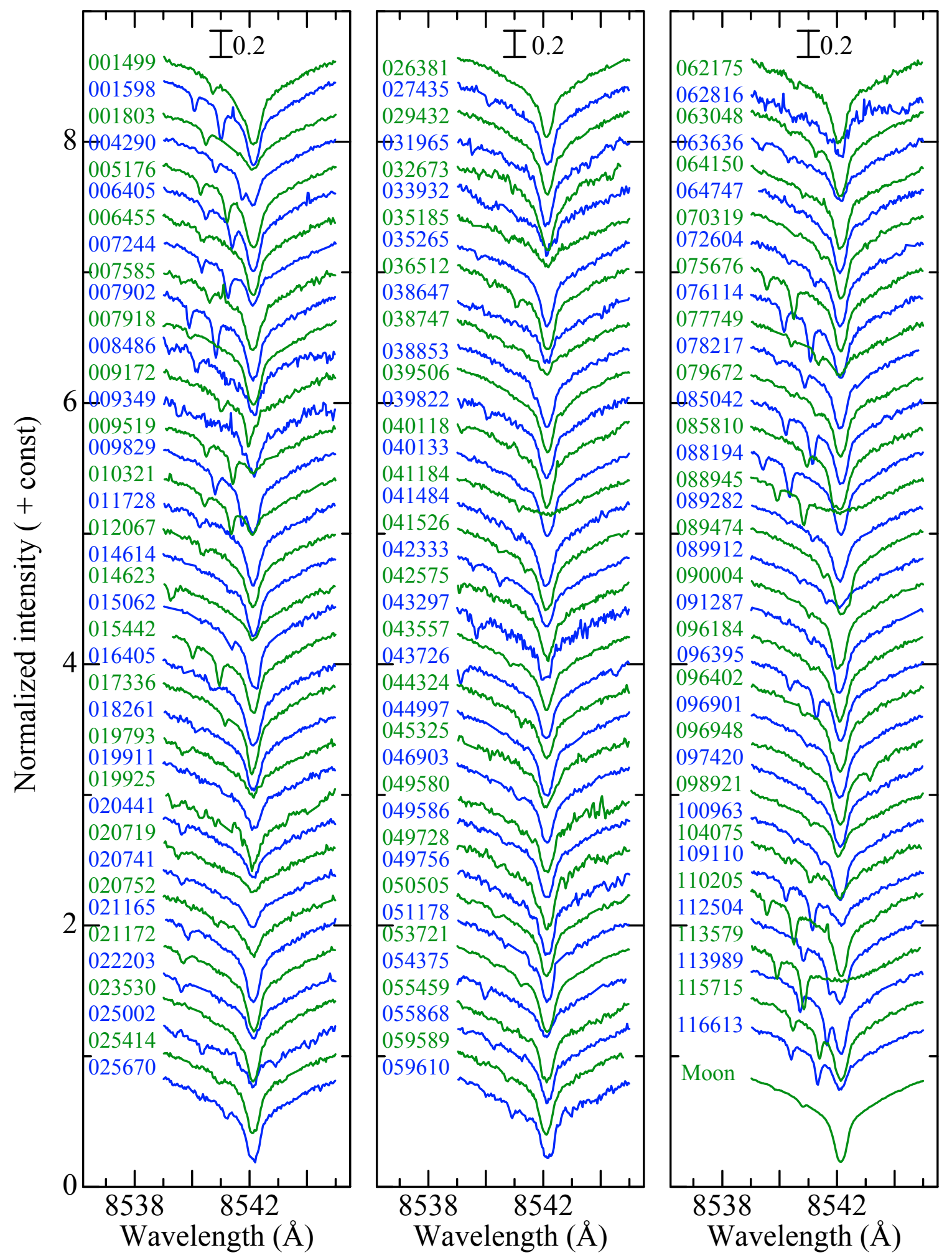

Fig. 2. Display of the Ca II 8542 line spectra for all the 118 program stars (along with the Moon/Sun). The wavelength scale of all stellar spectra is adjusted to the laboratory frame by correcting the radial velocity shifts. The HIP numbers are indicated in the figure.

where $v_{\mathrm{mt}}$ is the $e$-folding width of the Gaussian macroturbulence function, which is related to the radial-tangential macroturbulence dispersion, $\zeta_{\mathrm{RT}}$, as $v_{\mathrm{mt}} \simeq 0.42 \zeta_{\mathrm{RT}}$. Therefore, for a given $v_{\mathrm{r}+\mathrm{m}}$, the corresponding $v_{\mathrm{e}} \sin i$ can be derived if $v_{\mathrm{mt}}$ is known. The problem is, however, that an appropriate assignment of $v_{\mathrm{mt}}$ is not easy, because it depends on depth (i.e., decreasing with atmospheric height), as is well known for the solar atmosphere (see, e.g., Takeda 1995a). We thus proceeded as follows: We recall that $v_{\mathrm{r}+\mathrm{m}}$ was derived in Paper I by performing spectrum fitting of the 6080-6089 $\AA$ region. The strengths of conspicuous lines 


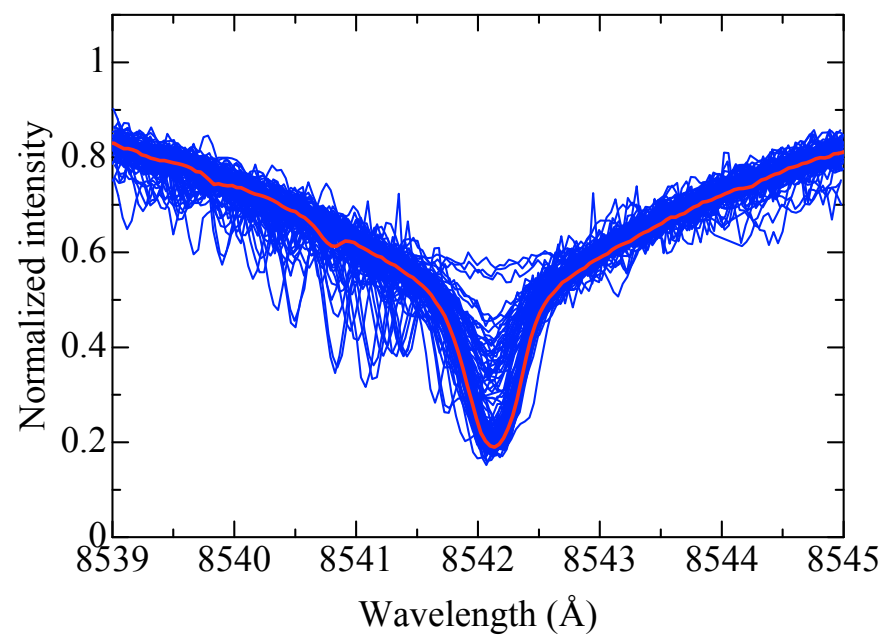

Fig. 3. Overplot of the core-region (within $\sim \pm 3 \AA$ from the line center) spectra of the Ca II 8542 line for all 118 stars, where the Moon spectrum is depicted in a thick (red) line. The wavelength scale of all stellar spectra is adjusted to the laboratory frame by correcting for the radial velocity shifts, by which telluric lines are placed at different positions from star to star (as can be recognized as jagged features, especially on the shorter wavelength side).

(those of $\mathrm{Ti}, \mathrm{Fe}, \mathrm{Ni}$ ) in these region are typically on the order of several tens $m \AA$, whose mean forming depths were estimated to be $\left\langle\log \tau_{5000}\right\rangle \sim-1$. With the help of Eq. (1) in Paper I, we may then adopt $v_{\mathrm{mt}} \simeq 1.5 \mathrm{~km} \mathrm{~s}^{-1}$ as a reasonable value of the Gaussian macroturbulence dispersion in the present case. Accordingly, we may derive $v_{\mathrm{e}} \sin i$ for each star as

$v_{\mathrm{e}} \sin i \simeq\left(v_{\mathrm{r}+\mathrm{m}}^{2}-1.5^{2}\right)^{1 / 2} / 0.94$.

Thus obtained $v_{\mathrm{e}} \sin i$ results are given in Table 1 .

While any high accuracy (e.g., compared to the case of detailed Fourier analysis of line profiles) cannot be expected in these $v_{\mathrm{e}} \sin i$ values, given the rough assumptions involved, we consider that they are surely of the correct order-of-magnitude and practically useful. Figure 4 shows the comparison of our $v_{\mathrm{e}} \sin i$ results with the data of Nordström et al. (2004) and Valenti \& Fisher (2005), from which we can recognize an almost reasonable consistency, even though some slight systematic trend of deviation is seen.

\section{Results and discussion}

\subsection{Rotation-lithium-activity connection}

The results of comparisons between $r_{0}(8542), v_{\mathrm{e}} \sin i$, and $A_{\mathrm{Li}}$ are depicted in Figs. 5a, d, and g, respectively.

In Fig. 5a, we can see that $r_{0}(8542)$ is closely correlated with $v_{\mathrm{e}} \sin i$ (i.e., chromospheric activity is enhanced with increasing rotational velocity), which matches the reasonable belief that activity is related to rotation-induced stellar dynamo. Figures $5 \mathrm{~d}$ and g clearly show evidence of the result we attempt here to prove: the surface $\mathrm{Li}$ content $\left(A_{\mathrm{Li}}\right)$ tends to decline with a decrease in stellar activity $\left(r_{0}\right)$ as well as in rotational rate $\left(v_{\mathrm{e}} \sin i\right)^{4}$. Since the comparatively higher rotator $\left(v_{\mathrm{e}} \sin i \gtrsim\right.$ $\left.5 \mathrm{~km} \mathrm{~s}^{-1}\right)$ with rather enhanced activity $\left(0.4 \lesssim r_{0} \lesssim 0.6\right)$

\footnotetext{
${ }^{4}$ We should remark that the residual intensity at the core gets more or less raised as $v_{\mathrm{e}} \sin i$ becomes higher due to the blurring effect (caused by correspondingly wider rotational broadening function being convolved). However, we can see from Fig. 5a (see the dotted line therein)
}

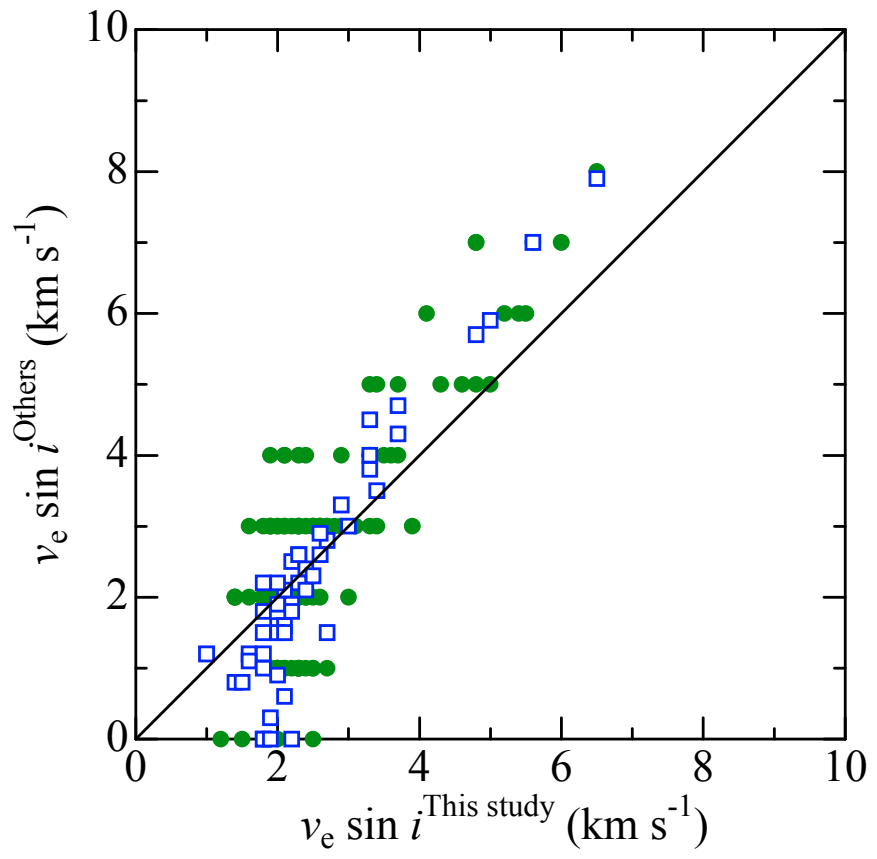

Fig. 4. Comparison of the $v_{\mathrm{e}} \sin i$ values evaluated in this study (abscissa) with those taken from the literature: Filled circles $\cdots$ Nordström et al. (2004) (for 111 stars in common), open squares ... Valenti \& Fisher (2005) (for 49 stars in common).

have $\mathrm{Li}$ abundances close to the solar-system value of $A_{\mathrm{Li}} \sim 3$, from which $A_{\mathrm{Li}}$ progressively decreases with decreasing $v_{\mathrm{e}} \sin i$ as well as $r_{0}(8542)$, we can confidently state that Li becomes increasingly depleted as the rotation is reduced in these solaranalog stars.

We should here recall that the rotational velocity (or angular momentum) is closely related to other parameters, such as the effective temperature ( $\left.T_{\text {eff }}\right)$ or the stellar age (age), because the deceleration of the rotation rate must be more effective for lower $T_{\text {eff }}$ stars with thicker convection zones, and older stars should have decelerated more than younger ones. As implied by Figs. 5 b, e, h ( $T_{\text {eff }}$-dependence) and Figs. 5 c,f, and i (age-dependence), such tendencies are recognized in the sense that rotational velocities tend to be decelerated more (or alternatively, activities as well as $\mathrm{Li}$ abundances tend to be lower) for older, cooler stars. However, since these trends are not so tight as those seen in the mutual correlations between $r_{0}(8542), v_{\mathrm{e}} \sin i$, and $A_{\mathrm{Li}}$ (Figs. 5a, d, and g), we may regard stellar rotation as the most important factor in controlling the surface $\mathrm{Li}$ abundance as well as the chromospheric activity among the various influential parameters.

\subsection{Angular momentum and envelope mixing}

We have thus seen that Li depletion in the surface of Sun-like stars progressively increases as stellar rotation becomes slower, as illustrated by the close correlation between $A_{\mathrm{Li}}$ and $r_{0}(8542)$ in addition to the $v_{\mathrm{e}} \sin i$-dependence of $r_{0}(8542)$. That planethost solar-analog stars tend to be Li-poor compared to their nonplanet-host counterparts, as first discovered by Israelian et al. (2004), may possibly be understood by, or at least partly related to, the difference in rotational properties between the two groups. This argument is supported by the finding of Gonzalez (2008),

that this effect is quantitatively insignificant compared to the main trend (symbols) which must thus be real. 

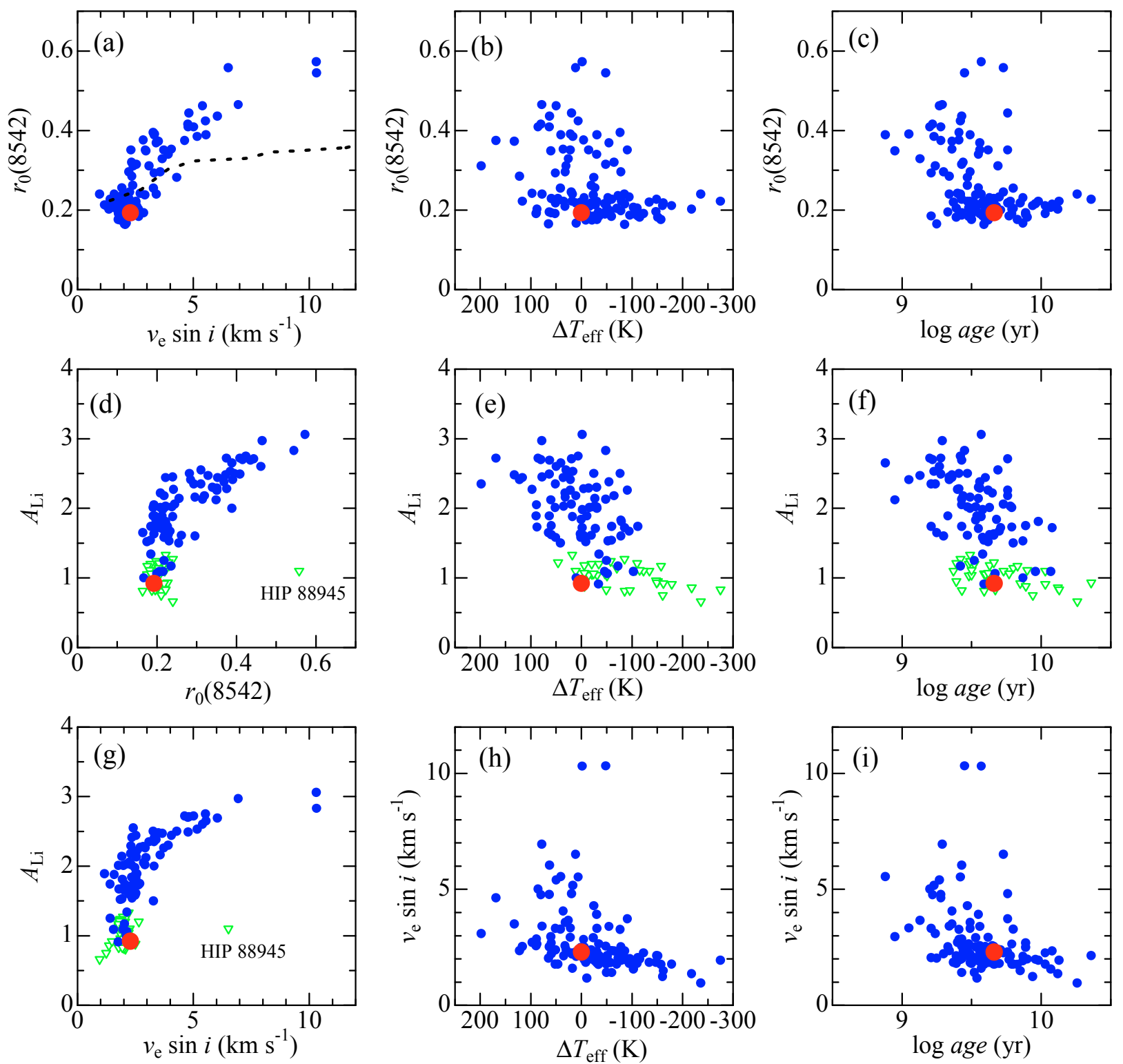

Fig. 5. Diagrams showing the correlation between the key physical parameters of $A_{\mathrm{Li}}, r_{0}(8542), v_{\mathrm{e}} \sin i, \log a g e$, and $\Delta T_{\text {eff }}$ (which are given in Table 1). a) $r_{0}(8542)$ vs. $v_{\mathrm{e}} \sin i$; b) $r_{0}(8542)$ vs. $\Delta T_{\mathrm{eff}}$; c) $r_{0}(8542)$ vs. $\log a g e ;$ d) $A_{\mathrm{Li}}$ vs. $r_{0}(8542)$; e) $A_{\mathrm{Li}}$ vs. $\Delta T_{\mathrm{eff}}$; f) $A_{\mathrm{Li}}$ vs. $\log a g e ;$ g) $A_{\mathrm{Li}}$ vs. $\left.v_{\mathrm{e}} \sin i, \mathbf{h}\right) v_{\mathrm{e}} \sin i$ vs. $\Delta T_{\mathrm{eff}}$; and $\left.\mathbf{i}\right) v_{\mathrm{e}} \sin i$ vs. $\log a g e$. The Sun (Moon) is indicated by the larger (red) circle. Regarding the panels involving $A_{\mathrm{Li}}$, the filled circles denote the combined results of well-reliable and less-reliable determinations (i.e., groups a) and $\mathbf{b}$ ) as described in Sect. 4.3 of Paper I), while the open inverse triangles show the upper limits for the unmeasurable cases. Note that panels e), f), and $\mathbf{g}$ ) are essentially equivalent to Fig. 11a, b, and 13b in Paper I, except that the latter show only the reliable (group (a)) values. The dotted line in panel a) represents the expected variation due to the blurring effect caused by an increase of $v_{\mathrm{e}} \sin i$ (simulated by convolving rotational broadening functions of various $v_{\mathrm{e}} \sin i$ values to the solar spectrum).

who reported (based on the literature data) that planet-harboring stars showing $\mathrm{Li}$ abundance anomalies (low $\mathrm{Li}$ ) also exhibit anomalies in terms of both $v_{\mathrm{e}} \sin i$ and $R_{\mathrm{HK}}^{\prime}$ (i.e., slow rotation and low activity).

In contrast, Israelian et al. (2009) could not find any meaningful Li vs. activity correlation or any tendency toward markedly small $v_{\mathrm{e}} \sin i$ for planet-harboring stars, while they confirmed the low-Li tendency of planet-host stars in a more convincing manner. We note, however, that their discussion is based on the data of stars with comparatively low- $v_{\mathrm{e}} \sin i$ $\left(\$ 3 \mathrm{~km} \mathrm{~s}^{-1}\right)$ as well as low-activity $\left(\log R_{\mathrm{HK}}^{\prime} \lesssim-4.7\right.$ which corresponds to $r_{0}(8542) \simeq 0.2$ according to Fig. 7) since their sample of planet-host stars are distributed across a limited parameter range (cf. their Figs. 2a, b). It is difficult to discuss any existence of parameter correlations in this low-rotation/activity region because of the progressive increase in the relative importance of errors. The relations that we have found between $A_{\mathrm{Li}}, v_{\mathrm{e}} \sin i$, and $r_{0}(8542)$ (being manifest when viewed over a rather wide range of these parameters; cf. Fig. 5a, d, g) would become appreciably unclear when we confine ourselves to this restricted region. Therefore, we can neither exclude nor accept their result based on their data alone (especially since they used imhomogeneous literature data of $v_{\mathrm{e}} \sin i$ and $R_{\mathrm{HK}}^{\prime}$ collected from several references). To settle this issue, far more precise evaluation of the stellar rotation rate as well as the stellar activity would be needed (e.g., direct determination of the rotation period by 
detecting the modulation of activity indicator based on long-term observations).

After recognizing that a lithium deficiency is more likely to take place in slower-rotation stars or planet-host stars, the next task is to find a reasonable explanation. Several possibilities for a rotation-mixing connection have been proposed, such as a turbulent diffusion mixing caused by magnetic rotational braking and an envelope mixing triggered by tidal forces from planets (see Gonzalez (2008) or Israelian et al. (2009) and the quoted references therein). Based on detailed theoretical simulations, Bouvier (2008) showed that slow rotators develop a high degree of differential rotation between the radiative core and the convective envelope, eventually promoting lithium depletion by enhanced mixing, while fast rotators experience little similar core-envelope decoupling. The Li-deficient tendency in planet-host stars may thus be caused by their slow rotation resulting from a long lasting star-disk interaction during the premain sequence phase. This line of theoretical approach should be pursued further. In any case, we should bear in mind that the observed evidence of $A_{\mathrm{Li}}-v_{\mathrm{e}} \sin i$ relation does not necessarily imply a direct physical connection, but may be the result of a long complex evolution involving different phases (such as the pre-main sequence).

\subsection{Sensitivity of Ca II 8542 as an activity index}

We point out problems that remain to be clarified. We are confident about the global rotation-activity-lithium relation in the regions of $3 \mathrm{~km} \mathrm{~s}^{-1} \lesssim v_{\mathrm{e}} \sin i \lesssim 10 \mathrm{~km} \mathrm{~s}^{-1}, 0.3 \lesssim r_{0}(8542) \lesssim 0.6$, and $2 \lesssim A_{\mathrm{Li}} \lesssim 3$, as shown in Figs. 5a, d, and g. However, little can be said about the comparatively low-rotation/activity/Li regions of $v_{\mathrm{e}} \sin i \lesssim 3 \mathrm{~km} \mathrm{~s}^{-1}, r_{0}(8542) \lesssim 0.3$, and $A_{\mathrm{Li}} \lesssim 2$, where $r_{0}(8542)$ tends to converge for the value of $\sim 0.2$, and $v_{\mathrm{e}} \sin i$ as well as $A_{\mathrm{Li}}$ are indefinite because they are close to the detection limit. This situation is apparent in the histograms of $r_{0}(8542)$, $v_{\mathrm{e}} \sin i$, and $A_{\mathrm{Li}}$ shown in Figs. 6a-c. How could this large dispersion in $A_{\mathrm{Li}}$ (of more than $\sim 1$ dex) be explained at $A_{\mathrm{Li}} \lesssim 2$, despite $r_{0}$ (8542) being stabilized at $\sim 0.2$ (cf. Fig. 2 d)?

In our opinion, this implies that the core flux of Ca II 8542 line is no longer a useful indicator; i.e., this line is too insensitive to any change in activity at this low-activity level. We confirmed by means of our non-LTE line-formation calculation (cf. Appendix B) that the core-flux of Ca II 8542 is not so sensitive to a mild chromospheric temperature rise (unless the temperature becomes sufficiently high to reach a certain threshold level; cf. Fig. B.1c), which makes itself rather unsuitable to studying the stellar activity at a lower level. This must be the reason for the convergence of $r_{0}(8542)$ at $\sim 0.2$.

In contrast, our non-LTE calculation suggested that the core emission of $\mathrm{Ca}$ II K line at $3934 \AA$ is sensitive to the chromospheric temperature enhancement of any degree (cf. Fig. B.1b), from which we may conclude that $\mathrm{Ca}$ II $\mathrm{H}+\mathrm{K}$ violet lines are more useful and practical (than $\mathrm{Ca}$ II near-IR triplet lines) at least for investigating the mild stellar activity of comparatively slow rotators. We can see from Fig. 7 that, while a reasonable correlation exists between $r_{0}(8542)$ and $\log R_{\mathrm{HK}}^{\prime}$ index (measure of the core emission strength of $\mathrm{Ca}$ II $\mathrm{H}+\mathrm{K}$ lines), $\log R_{\mathrm{HK}}^{\prime}$ still exhibits an appreciable dispersion around $\sim-5$, whereas $r_{0}(8542)$ stabilizes at $\sim 0.2$. This means that we still may have a chance to study the rotation-activity-lithium connection of comparatively slow rotators ( $v_{\mathrm{e}} \sin i \lesssim 3 \mathrm{~km} \mathrm{~s}^{-1}$, where Ca II IR triplet is no more effective) by studying the $\mathrm{Ca}$ II $\mathrm{H}+\mathrm{K}$ lines. Additional investigation
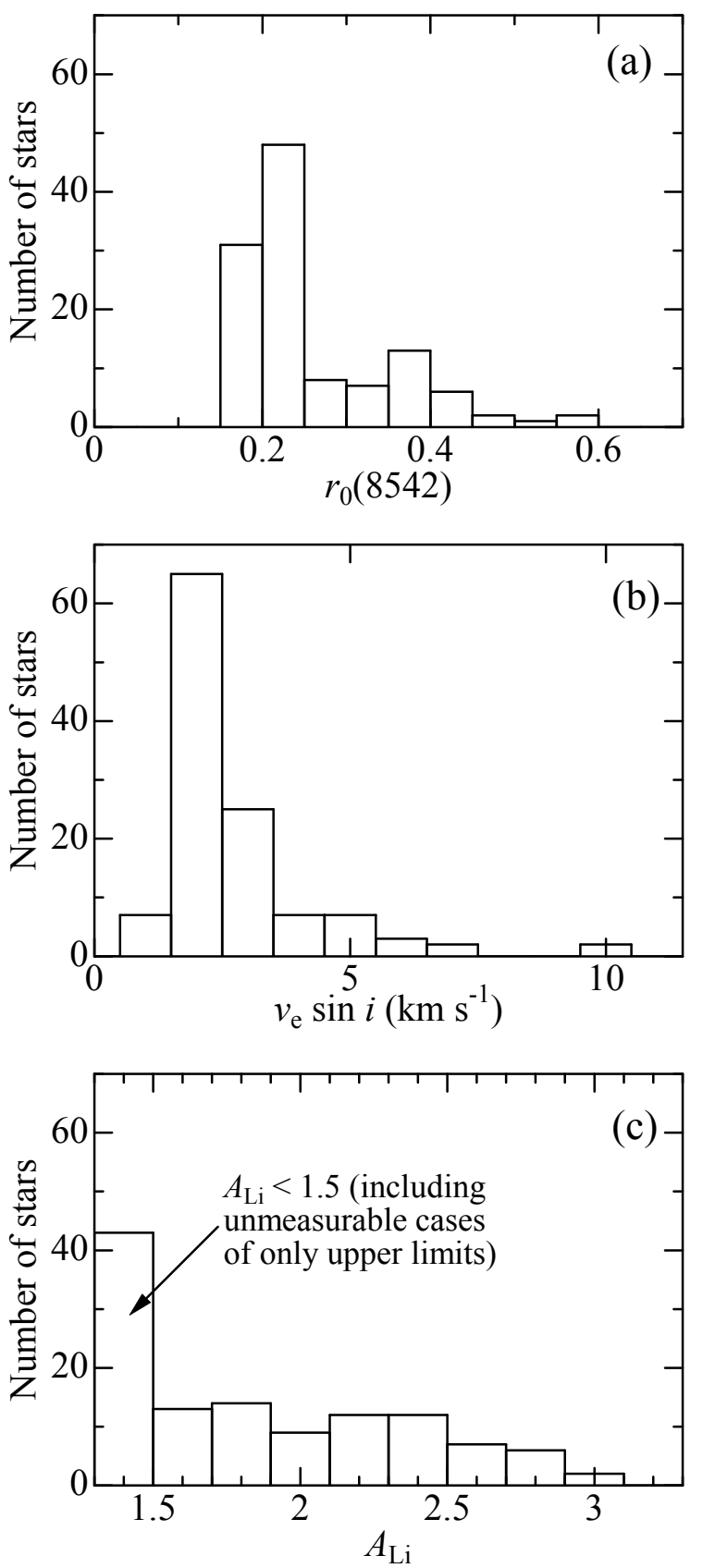

Fig. 6. Histograms showing the distributions of a) $r_{0}(8542)$; b) $v_{\mathrm{e}} \sin i$; and c) $A_{\mathrm{Li}}$, for our sample of 118 solar analogs.

along these lines would be worthwhile as we proceed to the next step.

\section{Conclusion}

In our previous study of Paper I, we carried out a comprehensive spectroscopic analysis of 118 solar analogs to clarify why the strengths of Li I 6708 line in these Sun-like stars are considerably diversified despite that they have stellar parameters quite similar to each other, and interestingly found a close relationship between the Li abundance and its line-width. We then proposed that stellar rotation may be the most important parameter in determining the surface Li content.

In this paper, we have tried to test this hypothesis by examining whether any correlation exists between the stellar 
Y. Takeda et al.: Li abundance, rotation, and stellar activity of solar analogs. II.

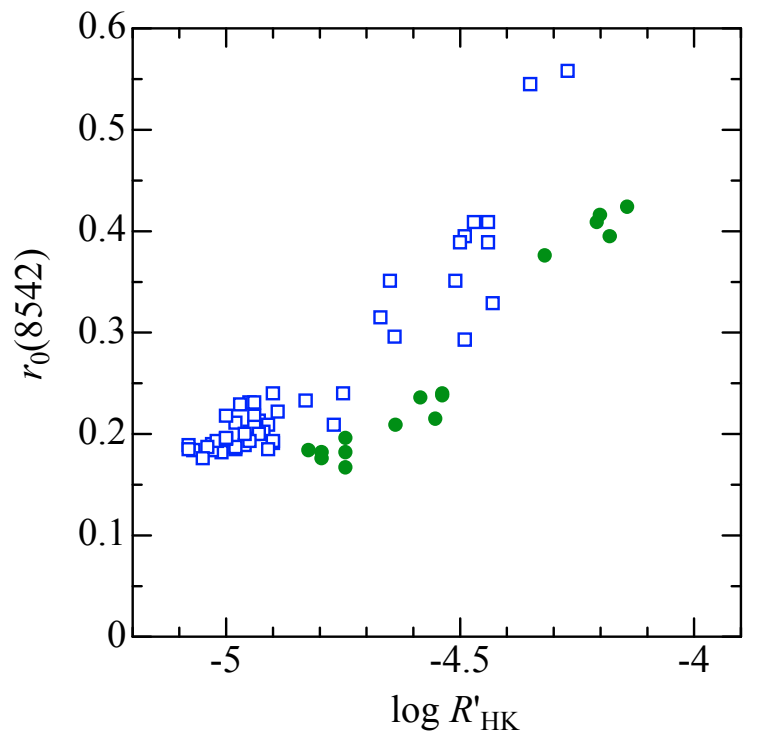

Fig. 7. Correlation of the $r_{0}(8452)$ values determined in this study with the $\log R_{\mathrm{HK}}^{\prime}$ indices taken from two papers: Filled circle $\cdots$ Strassmeier et al. (2000) (16 stars in common), open squares ... Wright et al. (2004) (50 stars in common).

activity and the Li abundance, as expected because of the widely believed rotation-activity connection. As an indicator of stellar activity, we used the residual line-center flux of the strong Ca II 8542 line $\left(r_{0}\right)$, which was measured from the high-dispersion near-IR spectra obtained with the $188 \mathrm{~cm}$ reflector and the HIDES spectrograph at Okayama Astrophysical Observatory. The projected rotational velocity $\left(v_{\mathrm{e}} \sin i\right)$ was reasonably accurately estimated by subtracting the contribution of the macroturbulence effect from the line-broadening width $\left(v_{\mathrm{r}+\mathrm{m}}\right)$ as we already established in Paper I.

Clear correlations have been confirmed in the diagrams $A_{\mathrm{Li}}$ vs. $r_{0}(8542), r_{0}(8542)$ vs. $v_{\mathrm{e}} \sin i$, and $A_{\mathrm{Li}}$ vs. $v_{\mathrm{e}} \sin i$, which support the arguments that (1) the stellar activity surely depends upon the rotational rate, and that (2) the atmospheric $\mathrm{Li}$ abundance of solar-analog stars declines progressively as the rotational velocity decreases.

We thus concluded that a Li-depletion mechanism in these Sun-like stars, most probably caused by effective envelope mixing, operates more efficiently as the stellar rotation slows down. In this context, it may be interesting/enlightening to interpret the observational finding of a low-Li tendency of planet-host $\mathrm{G}$ dwarfs within the framework of the rotational properties (i.e., difference in the angular momentum), as stated in the theoretical prediction by Bouvier (2008). Additional detailed investigations along those lines would be worthwhile.

However, the cause of this interconnection, which is found for comparatively high-rotation/activity/Li stars, remains unclear for the group of stars with low-rotation/activity/Li, where $r_{0}(8542)$ tends to converge and stabilize at $\sim 0.2$ and can no longer be a useful activity indicator. Since we found from our non-LTE calculation that Ca II H+K violet lines at 3968/3934 are more sensitive and useful (than Ca II IR triplet lines) for investigating the mild stellar activity of comparatively slow rotators, it would be beneficial to revisit this problem by studying these $\mathrm{Ca}$ II $\mathrm{H}+\mathrm{K}$ lines in greater detail.

Acknowledgements. We thank the staff of the Okayama Astrophysical Observatory for their kind and elaborate support in the observations. Constructive comments from an anonymous referee concerning the interpretation of low-Li tendency in planet-host stars are also acknowledged.
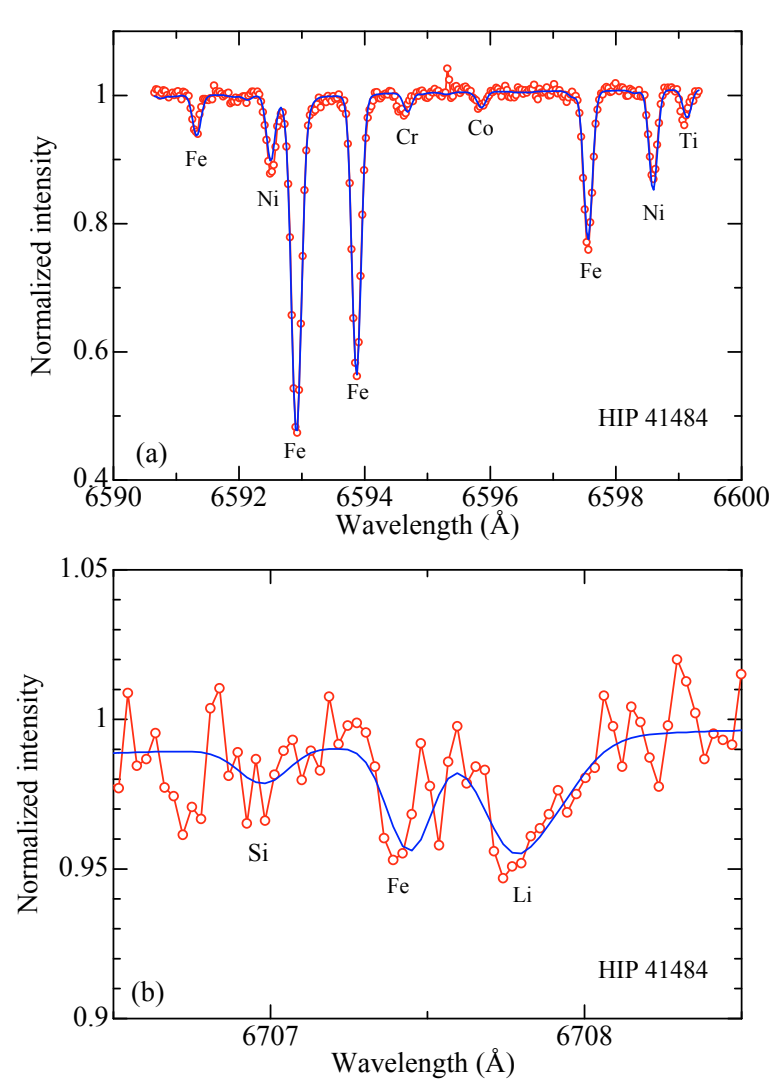

Fig. A.1. Spectrum fitting analysis applied to selected wavelength regions of HIP 41484. The observed and theoretical spectra are shown by open (line-connected) circles and solid lines, respectively. a) 6591-6599 ^ spectrum region including lines of $\mathrm{Ti}, \mathrm{Cr}, \mathrm{Fe}, \mathrm{Ni}$, and Co (for line-width evaluation); b) 6706.5-6708.5 Å region comprising lines of $\mathrm{Si}, \mathrm{Fe}$, and $\mathrm{Li}$ (for $\mathrm{Li}$ abundance determination).

\section{Appendix A: Reanalysis of HIP 41484}

In the course of this study, we noticed that the near-IR spectrum of HIP 41484 obtained for this study and the red-region spectrum used in Paper I were apparently inconsistent with each other. After careful inspection of the observational information (e.g., telescope pointing log), we then realized that the red spectrum obtained for this star in 2005 November 26 was not that of HIP 41484 but that of HIP 41184 (which was also one of our solar-analog targets); i.e., we had mistakenly observed the incorrect star because of the similarity in their HIP number ${ }^{5}$.

Since an erroneous spectrum was eventually used in Paper I, all the spectrum-related quantities derived therein were also wrong. We thus carried out an entire reanalysis of HIP 41484 based on the new spectrum used for this study, where a long wavelength range of 6300-10000 $\AA$ was fortunately available (thanks to the 3 mosaicked CCDs; cf. footnote 2) in addition to the main region of 7600-8800 $\AA$ including the Ca II triplet. The atmospheric parameters $\left(T_{\text {eff }}, \log g, v_{\mathrm{t}}\right.$, and $\left.[\mathrm{Fe} / \mathrm{H}]\right)$ were derived from the Fe I and Fe II lines 6300-7600 $\AA$ region. Since the 6080-6089 $\AA$ region used in Paper I to evaluate the linebroadening width by the spectrum-fitting method was not available in the present case, we instead used the 6591-6599 A region for this purpose (Fig. A.1a). The Li abundance was determined

\footnotetext{
5 As a result, HIP 41184 was unintentionally observed twice. In Figs. 6 and 8 in Paper I, the two spectra labeled as "41184" and "41484" actually correspond to the same star (HIP 41184).
} 
Table A.1. Redetermined stellar parameters and physical quantities of HIP 41484.

\begin{tabular}{|c|c|c|}
\hline $\mathrm{Sp}$ & G5V & Spectral type \\
\hline$T_{\mathrm{eff}}^{\mathrm{std}}(\mathrm{K})$ & 5864 & Effective temperature \\
\hline $\log g^{\mathrm{std}}\left(\mathrm{cm} \mathrm{s}^{-2}\right)$ & 4.33 & Surface gravity \\
\hline$v_{\mathrm{t}}^{\mathrm{std}}\left(\mathrm{km} \mathrm{s}^{-1}\right)$ & 0.92 & Microturbulence \\
\hline$\{\mathrm{Fe} / \mathrm{H}\}$ & +0.05 & Metallicity $\left(\equiv A_{\mathrm{Fe}}-7.50\right)$ \\
\hline$V$ & 6.32 & Apparent visual magnitude \\
\hline$p$ (mas) & $45.89( \pm 0.84)$ & Hipparcos parallax \\
\hline$M_{V}$ & 4.63 & Absolute visual magnitude \\
\hline B.C. & -0.12 & Bolometric correction \\
\hline$M_{\text {bol }}$ & 4.51 & Absolute bolometric magnitude \\
\hline $\log \left(L / L_{\text {sun }}\right)$ & 0.096 & Bolometric luminosity \\
\hline$M_{\text {iso }} / M_{\text {sun }}$ & $1.06_{-0.03}^{+0.04}$ & Mass (from tracks) \\
\hline $\log a g e_{\text {iso }}(\mathrm{yr})$ & $9.37_{-0.40}^{+0.29}$ & Age (from tracks) \\
\hline$M_{\text {iso }} / M_{\text {sun }}$ & $1.05_{-0.05}^{+0.05}$ & Mass (from isochrones) \\
\hline $\log a g e_{\mathrm{iso}}(\mathrm{yr})$ & $9.54_{-0.34}^{+0.32}$ & Age (from isochrones) \\
\hline$\Delta T_{\mathrm{eff}}(\mathrm{K})$ & +87.6 & Star-Sun $T_{\text {eff }}$ \\
\hline$\Delta \log g\left(\mathrm{~cm} \mathrm{~s}^{-2}\right)$ & -0.036 & Star-Sun $\log g$ \\
\hline$\Delta v_{\mathrm{t}}\left(\mathrm{km} \mathrm{s}^{-1}\right)$ & -0.10 & Star-Sun $v_{\mathrm{t}}$ \\
\hline$\Delta\{\mathrm{Fe} / \mathrm{H}\}$ & +0.060 & Star-Sun metallicity $([\mathrm{Fe} / \mathrm{H}])$ \\
\hline$V_{\text {hel }}\left(\mathrm{km} \mathrm{s}^{-1}\right)$ & -32.3 & Heliocentric radial velocity \\
\hline$\left\langle R_{\mathrm{m}}\right\rangle(\mathrm{kpc})$ & 7.337 & Mean galactocentric distance \\
\hline$e$ & 0.136 & Orbital eccentricity \\
\hline$z_{\max }(\mathrm{kpc})$ & 0.172 & Max. separation from galactic plane \\
\hline$U_{\mathrm{lsr}}\left(\mathrm{km} \mathrm{s}^{-1}\right)$ & +30.5 & Space velocity (radial) \\
\hline$V_{\mathrm{lsr}}\left(\mathrm{km} \mathrm{s}^{-1}\right)$ & -23.4 & Space velocity (tangential) \\
\hline$W_{\mathrm{lsr}}\left(\mathrm{km} \mathrm{s}^{-1}\right)$ & -15.2 & Space velocity (vertical) \\
\hline$E W(6708)(\mathrm{mÅ})$ & 11.0 & Equivalent width of Li I 6708 \\
\hline$A_{\mathrm{Li}}(\mathrm{NLTE})$ & 1.73 & Non-LTE Li abundance \\
\hline$\Delta_{\mathrm{NLTE}}$ & +0.06 & Non-LTE correction \\
\hline$v_{\mathrm{r}+\mathrm{m}}\left(\mathrm{km} \mathrm{s}^{-1}\right)$ & 2.90 & Macrobroadening velocity \\
\hline$v_{\mathrm{e}} \sin i\left(\mathrm{~km} \mathrm{~s}^{-1}\right)$ & 2.64 & Projected rotational velocity \\
\hline
\end{tabular}

Notes. These are the revised parameters of HIP 41484 established based on the new spectrum data for this star, since the values of spectroscopically determined quantities (e.g., atmospheric as well as linebroadening parameters, radial velocity, and $\mathrm{Li}$ abundance) for this star reported in Paper I turned out to be erroneous because a wrong spectrum (actually that of HIP 41184) was used. See Paper I for more details of the meanings of these quantities and the procedures for their determinations.

from the Li I doublet at $6708 \AA$ (Fig. A.1b). Otherwise, all the relevant stellar parameters were established in the same way as in Paper I. The results of this reanalysis are summarized in Table A.1.

\section{Appendix B: Ca II $\mathrm{H}+\mathrm{K}$ doublet and near-IR triplet as stellar-activity indicators}

It is well known that the cores of strong Ca II lines, such as the resonance $\mathrm{H}+\mathrm{K}$ doublet lines (at 3968 and $3934 \AA$ ) or the nearIR triplet lines (at 8498/8542/8662 $\AA$ ), reflect the temperature structure of the upper atmosphere and may be used as useful indicators of chromospheric activity. However, does any difference exist between these two activity indicators in their practical applications; do they have any specific strong or weak points depending on situations? To answer this, we tried to compute the profiles of Ca II 3934 and Ca II 8542 lines for several test model atmospheres with different chromospheric effects, and examine how the core fluxes of these two lines respond to the temperature profile of upper atmospheres.

We must take into account non-LTE effects, because the dilution of the line source function in the upper optically-thin layer determines the intensity/flux level at the core. Non-LTE statistical-equilibrium calculations directed specifically toward studying the formation of these strong $\mathrm{Ca}$ II lines in the chromosphere have been few in number ${ }^{6}$. Except for the pioneering work on a simple 3-level Ca II model ion (e.g., Linsky \& Avrett 1970 for the H+K lines; Linsky et al. 1979 for the 8542 line), the only relevant non-LTE study quotable here may be, to our knowledge, that of Andretta et al. (2005), who computed (based on a model atom comprising $18 \mathrm{Ca}$ I and $5 \mathrm{Ca}$ II levels) the $8498 / 8542 / 8662$ line profiles for various solar models including a semi-empirical one with the chromosphere.

We dealt with this problem here using a more detailed atomic model of calcium (111/50 terms and 2376/313 radiative transitions for $\mathrm{Ca}$ I/Ca II), comprising up to $\mathrm{Ca}$ I 4 s $16 \mathrm{~d}^{3} \mathrm{D}$ (48830 $\mathrm{cm}^{-1}$ from the ground level) and Ca II $3 p^{6} 16 d^{2} \mathrm{D}$ (93895 $\mathrm{cm}^{-1}$ from the ground level), which was constructed from the atomic-line database compiled by Kurucz \& Bell (1995). The electron collision cross-sections relevant to the lowest 7 terms of Ca II were taken from Burgess et al. (1995). The data from TOPbase (Cunto \& Mendoza 1992) were adopted for the photoionization cross-sections for the lowest 7 and 10 terms of Ca I and Ca II, respectively. As for other computational details (e.g., electron-collision rates as well as photoionization rates for the remaining terms not mentioned above, collisional ionization rates, treatment of collisions with neutral-hydrogen atoms), we followed the recipe described in Sect. 3.1.3 of Takeda (1991).

We tested three solar atmospheric models $\left(T_{\text {eff }}=5780 \mathrm{~K}\right.$, $\log g=4.44,[\mathrm{Fe} / \mathrm{H}]=0.0)$ that have different temperature profiles only at the upper layer of $\tau_{5000} \lesssim 10^{-4}$. Model $\mathrm{C}$ has a chromospheric temperature structure similar to that of the semiempirical solar model of Maltby et al. (1986). Model E is equivalent to Kurucz's (1979) ATLAS6 solar model (without any temperature rise), and Model M corresponds to the mean of these two (cf. Fig. B.1a). The pressure/density structures of these models were obtained by integrating the equation of hydrostatic equilibrium. We refer to Sect. 2.1 of Takeda (1995b) for more details about how Models C and E were constructed. We applied a depth-dependent microturbulence by adopting the turbulent velocity fields given in Table 11 of Maltby et al. (1986).

The resulting profiles of $\mathrm{Ca}$ II 3934 and 8542 lines are depicted in Figs. B.1b, c, respectively, where the corresponding solar flux spectra of Kurucz et al. (1984) are also shown for comparison. It can be seen that the computed profiles for Model $\mathrm{C}$, which is likely to be the most realistic among the three, do not reproduce the true solar spectra well (i.e., the core flux level is too high), indicating that our modeling is still imperfect. However, our intention here is not to accomplish excellent fitting between theory and observation, but to ascertain/determine whether and how the sensitivity to the upper temperature differs between these two lines. From this standpoint, we can recognize an important result in these figures: The strength of the core emission in the Ca II 3934 line progressively increases in accordance with the temperature rise in the upper

\footnotetext{
6 More precisely, several other non-LTE studies of calcium lines in late-type stars (e.g., Watanabe \& Steenbock 1985; Drake 1991; Jørgensen et al. 1992; Mashonkina et al. 2007), focusing mainly on non-LTE abundance corrections, do not explicitly address the chromospheric effect (core emission) on the formation of these activitysensitive Ca II lines under question.
} 
Y. Takeda et al.: Li abundance, rotation, and stellar activity of solar analogs. II.
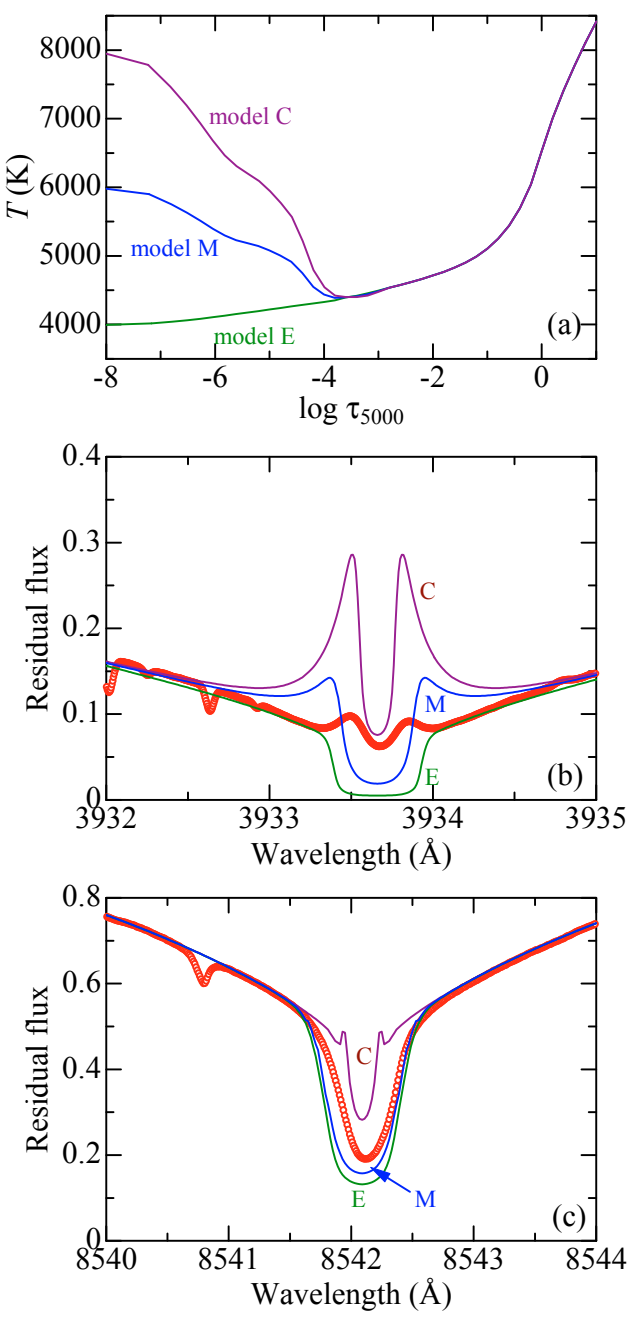

Fig. B.1. Test simulations for the core profiles of Ca II 3934 (K) line and $\mathrm{Ca}$ II 8542 line, based on the non-LTE calculations carried out on three model atmospheres (Models $\mathrm{C}, \mathrm{M}$, and $\mathrm{E}$ ) with different temperature structures at the upper atmosphere. a) Temperature profiles of Models C, M, and E. b) Simulated (flux) profiles of the Ca II 3934 line; c) simulated (flux) profiles of the Ca II 8542 line. In panels b) and c), Kurucz et al.'s (1984) solar flux spectra are also indicated by open circles.

layer as Model $\mathrm{E} \rightarrow$ Model $\mathrm{M} \rightarrow$ Model C (Fig. B.1b). In contrast, the residual core flux of Ca I 8542 line barely differs for Models $\mathrm{E}$ and $\mathrm{M}$, while that for Model $\mathrm{C}$ is appreciably higher
(Fig. B.1c), which means that this indicator is not very useful for studying the moderate temperature enhancement (mild chromospheric activity) because of its rather inefficient response until the temperature rise goes over a certain threshold level. Consequently, we may conclude that those who intend to study the nature of comparatively mild stellar activity in slower rotators should use the Ca II $\mathrm{H}+\mathrm{K}$ lines at $3934 / 3968 \AA$, rather than the Ca II triplet lines at 8498/8542/8662 ̊.

\section{References}

Andretta, V., Busà, I., Gomez, M. T., et al. 2005, A\&A, 430, 669 Bouvier, J. 2008, A\&A, 489, L53

Burgess, A., Chidichimo, M. C., \& Tully, J. A. 1995, A\&A, 300, 627

Busà, I., Aznar Cuadrado, R., Terranegra, L., Andretta, V., \& Gomez, M. T. 2007, A\&A, 466, 1089

Chmielewski, Y. 2000, A\&A, 353, 666

Cunto, W., \& Mendoza, C. 1992, Rev. Mex. Astron. Astrofis., 23, 107

Drake, J. J. 1991, MNRAS, 251, 369

Foing, B. H., Crivellari, L., Vladilo, G., Rebolo, R., \& Beckman, J. E. 1989, A\&AS, 80, 189

Gonzalez, G. 2008, MNRAS, 386, 928

Israelian, G., Santos, N. C., Mayor, M., et al. 2004, A\&A, 414, 601

Israelian, G., Delgado Mena, E., Santos, N. C., et al. 2009, Nature, 462, 189

Izumiura, H. 1999, in Proc. 4th East Asian Meeting on Astronomy, Observational Astrophysics in Asia and its Future ed. P. S. Chen (Kunming: Yunnan Observatory), 77

Jørgensen, U. G., Carlsson, M., \& Johnson, H. R. 1992, A\&A, 254, 258

Kurucz, R. L. 1979, ApJS, 40, 1

Kurucz, R. L., \& Bell, B. 1995, Kurucz CD-ROM, No. 23 (Harvard-Smithsonian Center for Astrophysics) [also available at http://kurucz .harvard.edu/ LINELISTS . html]

Kurucz, R. L., Furenlid, I., Brault, J., et al. 1984, Solar Flux Atlas from 296 to $1300 \mathrm{~nm}$ (Sunspot, New Mexico: National Solar Observatory) [digital version available at http://kurucz.harvard.edu/sun.html]

Linsky, J. L., \& Avrett, E. H. 1970, PASP, 82, 169

Linsky, J. L., Hunten, D. M., Sowell, R., Glackin, D. L., \& Kelch, W. L. 1979, ApJS, 41, 481

Mallik, S. V. 1997, A\&AS, 124, 359

Maltby, P., Avrett, E. H., Carlsson, M., et al. 1986, ApJ, 306, 284

Mashonkina, L., Korn, A. J., \& Przybilla, N. 2007, A\&A, 461, 261

Nordström, B., Mayor, M., Andersen, J., et al. 2004, A\&A, 418, 989

Strassmeier, K., Washuettl, A., Granzer, Th., Scheck, M., \& Weber, M. 2000, A\&AS, 142, 275

Takeda, Y. 1991, A\&A, 242, 455

Takeda, Y. 1995a, PASJ, 47, 337

Takeda, Y. 1995b, PASJ, 47, 463

Takeda, Y., \& Tajitsu, A. 2009, PASJ, 61, 471

Takeda, Y., Kawanomoto, S., Honda, S., Ando, H., \& Sakurai, T. 2007, A\&A, 468, 663 (Paper I)

Valenti, J. A., \& Fisher, D. A. 2005, ApJS, 159, 141

Watanabe, T., \& Steenbock, W. 1985, A\&A, 149, 21

Wright, J. T., Marcy, G. W., Butler, R. P., et al. 2004, ApJS, 152, 261 
A\&A 515, A93 (2010)

Table 1. Activity index, rotation, Li abundance, age, and the atmospheric parameters.

\begin{tabular}{|c|c|c|c|c|c|c|c|c|c|c|}
\hline HIP & $r_{0}(8542)$ & $v_{\mathrm{e}} \sin i$ & $A_{\mathrm{Li}}$ & log age & $\Delta T_{\text {eff }}$ & $\Delta \log g$ & $\Delta v_{\mathrm{t}}$ & $\Delta\{\mathrm{Fe} / \mathrm{H}\}$ & Date & Remark \\
\hline 001499 & 0.190 & 2.01 & $(<1.1)$ & 9.59 & -46.7 & -0.08 & -0.04 & +0.198 & $2008-08-11$ & \\
\hline 001598 & 0.222 & 1.94 & 1.81 & 9.98 & -79.2 & -0.14 & -0.05 & -0.274 & 2008-08-11 & \\
\hline 001803 & 0.389 & 5.55 & 2.65 & 8.88 & +40.6 & -0.08 & +0.19 & +0.230 & 2008-08-11 & \\
\hline 004290 & 0.320 & 2.34 & 2.18 & 9.44 & -64.7 & -0.08 & +0.12 & -0.129 & 2008-08-12 & \\
\hline 005176 & 0.195 & 2.52 & 1.89 & 9.33 & +63.4 & -0.12 & +0.06 & +0.166 & $2008-08-15$ & \\
\hline 006405 & 0.213 & 2.07 & 1.71 & 9.55 & -27.9 & -0.07 & -0.01 & -0.133 & $2008-08-12$ & \\
\hline 006455 & 0.227 & 1.41 & 1.74 & 9.43 & -60.1 & +0.05 & +0.00 & -0.089 & $2008-12-02$ & \\
\hline 007244 & 0.351 & 2.31 & 2.29 & 9.56 & -14.3 & +0.07 & +0.11 & -0.036 & 2008-08-14 & \\
\hline 007585 & 0.209 & 2.34 & 1.84 & 9.68 & +0.2 & -0.01 & +0.04 & +0.067 & $2007-02-05$ & \\
\hline 007902 & 0.182 & 2.13 & $(<0.9)$ & 9.39 & -151.0 & -0.07 & -0.10 & +0.004 & 2008-08-13 & \\
\hline 007918 & 0.191 & 2.57 & 1.89 & 9.56 & +89.5 & -0.12 & +0.12 & +0.025 & 2007-02-05 & \\
\hline 008486 & 0.311 & 2.41 & 2.55 & 9.69 & +30.2 & +0.00 & +0.09 & -0.055 & 2007-02-06 & \\
\hline 009172 & 0.282 & 4.29 & 2.50 & 9.47 & -24.8 & +0.06 & +0.16 & +0.040 & $2008-08-13$ & \\
\hline 009349 & 0.245 & 2.26 & 2.06 & 9.73 & +19.7 & -0.06 & +0.03 & +0.012 & 2007-02-07 & \\
\hline 009519 & 0.465 & 6.94 & 2.97 & 9.29 & +78.0 & -0.03 & +0.20 & +0.139 & $2008-08-15$ & \\
\hline 009829 & 0.211 & 1.77 & $(<0.9)$ & 10.03 & -178.6 & -0.16 & -0.06 & -0.295 & $2008-08-13$ & \\
\hline 010321 & 0.395 & 3.27 & 2.50 & 9.41 & -76.6 & +0.09 & +0.09 & -0.026 & 2008-08-15 & \\
\hline 011728 & 0.205 & 2.11 & $(<1.0)$ & 9.48 & -50.7 & -0.05 & +0.01 & +0.033 & 2008-12-03 & \\
\hline 012067 & 0.240 & 1.97 & $(<1.3)$ & 9.42 & -85.3 & -0.06 & -0.09 & +0.197 & $2008-12-02$ & \\
\hline 014614 & 0.220 & 2.49 & 1.58 & 9.58 & +1.2 & -0.09 & +0.03 & -0.102 & 2008-12-01 & \\
\hline 014623 & 0.389 & 3.31 & 2.00 & 9.49 & -30.8 & +0.01 & +0.09 & +0.116 & $2008-12-02$ & \\
\hline 015062 & 0.222 & 2.32 & 2.02 & 9.47 & -30.6 & +0.03 & -0.08 & -0.276 & 2008-08-14 & \\
\hline 015442 & 0.233 & 1.80 & 1.67 & 9.70 & -94.5 & +0.03 & -0.10 & -0.200 & 2008-08-14 & \\
\hline 016405 & 0.184 & 2.65 & $(<1.2)$ & 9.48 & -34.4 & -0.15 & +0.02 & +0.264 & 2008-12-02 & \\
\hline 017336 & 0.164 & 2.07 & $(<0.8)$ & 9.59 & -85.5 & +0.09 & -0.06 & -0.119 & 2008-08-14 & \\
\hline 018261 & 0.242 & 2.71 & 2.27 & 9.40 & +97.4 & -0.04 & +0.01 & +0.013 & 2008-12-02 & \\
\hline 019793 & 0.385 & 5.17 & 2.53 & 9.23 & +16.7 & -0.03 & +0.25 & +0.165 & $2008-12-02$ & \\
\hline 019911 & 0.351 & 3.73 & 2.26 & 9.76 & -91.1 & -0.12 & +0.14 & -0.133 & 2008-12-03 & \\
\hline 019925 & 0.262 & 2.39 & 1.61 & 9.47 & -19.5 & +0.04 & -0.01 & +0.064 & 2007-02-07 & \\
\hline 020441 & 0.376 & 2.84 & 2.28 & 9.41 & -4.5 & -0.02 & +0.03 & +0.136 & $2008-12-03$ & \\
\hline 020719 & 0.462 & 5.40 & 2.60 & 9.27 & +49.8 & -0.13 & +0.23 & +0.128 & 2008-12-03 & \\
\hline 020741 & 0.391 & 3.33 & 2.41 & 9.05 & +21.7 & -0.11 & +0.18 & +0.164 & 2008-12-03 & \\
\hline 020752 & 0.375 & 4.63 & 2.72 & 9.28 & +168.9 & -0.01 & +0.16 & +0.163 & $2008-12-02$ & \\
\hline 021165 & 0.220 & 2.53 & 1.61 & 9.66 & -4.5 & -0.20 & +0.03 & -0.164 & $2008-12-03$ & \\
\hline 021172 & 0.196 & 1.85 & $(<1.0)$ & 9.63 & -147.4 & -0.15 & -0.08 & -0.110 & 2008-12-02 & \\
\hline 022203 & 0.340 & 3.92 & 2.30 & 9.55 & -30.4 & -0.17 & +0.12 & +0.123 & $2008-12-03$ & \\
\hline 023530 & 0.211 & 1.24 & $(<0.8)$ & 9.94 & -160.7 & -0.08 & -0.06 & -0.238 & 2008-12-01 & \\
\hline 025002 & 0.369 & 3.40 & 2.38 & 9.56 & -54.6 & -0.03 & +0.09 & -0.096 & 2007-02-07 & \\
\hline 025414 & 0.220 & 1.85 & $(<1.1)$ & 9.37 & -134.4 & +0.05 & -0.11 & +0.095 & 2008-12-01 & \\
\hline 025670 & 0.218 & 2.05 & $(<1.2)$ & 9.52 & -20.1 & +0.06 & -0.10 & +0.090 & 2008-12-02 & \\
\hline 026381 & 0.240 & 0.96 & $(<0.7)$ & 10.26 & -236.2 & +0.03 & -0.08 & -0.442 & 2008-12-01 & planet-host star \\
\hline 027435 & 0.231 & 1.92 & 1.53 & 9.87 & -52.6 & +0.02 & -0.06 & -0.204 & $2008-12-03$ & \\
\hline 029432 & 0.200 & 2.14 & 1.06 & 9.67 & -51.4 & -0.06 & +0.00 & -0.111 & $2008-12-03$ & \\
\hline 031965 & 0.167 & 2.11 & 1.00 & 9.87 & +10.2 & -0.11 & +0.01 & +0.051 & 2007-02-05 & \\
\hline 032673 & 0.176 & 1.83 & $(<1.0)$ & 9.50 & -30.9 & +0.14 & -0.07 & +0.075 & 2007-02-05 & \\
\hline 033932 & 0.373 & 3.50 & 2.48 & 9.36 & +132.4 & -0.07 & +0.11 & -0.112 & $2008-12-05$ & \\
\hline 035185 & 0.444 & 4.81 & 2.71 & 9.76 & +19.5 & -0.23 & +0.27 & -0.003 & $2008-12-01$ & \\
\hline 035265 & 0.191 & 1.77 & 2.01 & 9.79 & +51.8 & -0.05 & +0.06 & -0.009 & 2007-02-05 & \\
\hline 036512 & 0.218 & 1.42 & 1.25 & 9.52 & -49.8 & +0.03 & -0.12 & -0.085 & 2008-12-03 & \\
\hline 038647 & 0.315 & 2.55 & 2.13 & 9.43 & -48.3 & -0.03 & -0.03 & +0.012 & $2007-02-05$ & \\
\hline 038747 & 0.424 & 5.53 & 2.75 & 9.42 & +6.3 & -0.07 & +0.11 & +0.025 & 2008-12-02 & \\
\hline 038853 & 0.222 & 2.53 & 2.44 & 9.60 & +116.6 & -0.22 & +0.06 & -0.064 & 2008-12-02 & \\
\hline 039506 & 0.227 & 2.14 & $(<0.9)$ & 10.36 & -148.9 & -0.14 & -0.16 & -0.610 & 2008-12-01 & \\
\hline 039822 & 0.231 & 1.97 & $(<1.2)$ & 9.63 & -5.6 & -0.11 & -0.08 & -0.215 & $2008-12-03$ & \\
\hline 040118 & 0.202 & 1.36 & $(<0.9)$ & 10.12 & -218.0 & -0.01 & -0.13 & -0.420 & 2007-04-19 & \\
\hline 040133 & 0.184 & 2.36 & 1.54 & 9.59 & -59.6 & -0.11 & -0.03 & +0.127 & 2008-12-01 & \\
\hline 041184 & 0.545 & 10.32 & 2.83 & 9.45 & -48.4 & +0.00 & +0.54 & +0.105 & $2008-12-01$ & \\
\hline 041484 & 0.202 & 2.64 & 1.73 & 9.79 & +87.6 & -0.04 & -0.10 & +0.060 & $2008-05-21$ & cf. Appendix A \\
\hline 041526 & 0.222 & 2.13 & 2.03 & 9.78 & +34.9 & -0.16 & -0.01 & -0.018 & 2008-12-03 & \\
\hline 042333 & 0.293 & 3.31 & 2.35 & 9.21 & +51.2 & +0.04 & +0.05 & +0.153 & $2008-12-03$ & \\
\hline 042575 & 0.236 & 2.03 & 1.17 & 9.42 & -72.6 & -0.02 & -0.04 & +0.073 & 2008-12-05 & \\
\hline 043297 & 0.296 & 2.22 & 1.60 & 9.30 & -78.3 & +0.01 & +0.05 & +0.081 & 2008-05-27 & \\
\hline 043557 & 0.255 & 3.28 & 1.50 & 9.80 & +41.2 & -0.03 & +0.05 & -0.061 & 2007-02-05 & \\
\hline 043726 & 0.240 & 1.59 & 1.88 & 9.57 & +17.5 & +0.04 & +0.01 & +0.125 & $2008-05-26$ & \\
\hline 044324 & 0.285 & 2.35 & 2.41 & 9.34 & +122.2 & +0.04 & +0.09 & -0.008 & 2008-12-03 & \\
\hline
\end{tabular}


Y. Takeda et al.: Li abundance, rotation, and stellar activity of solar analogs. II.

Table 1. continued.

\begin{tabular}{|c|c|c|c|c|c|c|c|c|c|c|}
\hline HIP & $r_{0}(8542)$ & $v_{\mathrm{e}} \sin i$ & $A_{\mathrm{Li}}$ & log age & $\Delta T_{\text {eff }}$ & $\Delta \log g$ & $\Delta v_{\mathrm{t}}$ & $\Delta\{\mathrm{Fe} / \mathrm{H}\}$ & Date & Remark \\
\hline 044997 & 0.198 & 1.84 & $(<1.2)$ & 9.49 & -57.8 & +0.14 & -0.28 & +0.055 & $2008-12-02$ & \\
\hline 045325 & 0.311 & 3.09 & 2.35 & 9.24 & +197.8 & +0.07 & +0.03 & +0.184 & 2008-12-05 & \\
\hline 046903 & 0.238 & 2.91 & 2.02 & 9.53 & -23.8 & -0.04 & +0.12 & -0.031 & 2008-12-02 & \\
\hline 049580 & 0.215 & 2.48 & 1.98 & 9.58 & -2.0 & -0.08 & -0.12 & +0.003 & 2007-02-07 & \\
\hline 049586 & 0.222 & 2.21 & $(<1.3)$ & 9.49 & +18.1 & -0.09 & +0.04 & +0.204 & 2008-12-02 & \\
\hline 049728 & 0.175 & 2.01 & $(<1.0)$ & 9.60 & -21.1 & -0.04 & -0.02 & -0.064 & 2007-02-06 & \\
\hline 049756 & 0.185 & 2.14 & 1.34 & 9.58 & -35.1 & -0.14 & +0.01 & +0.027 & 2007-02-06 & \\
\hline 050505 & 0.218 & 1.49 & $(<0.9)$ & 9.85 & -162.1 & -0.01 & -0.11 & -0.165 & 2007-04-19 & \\
\hline 051178 & 0.229 & 1.77 & $(<1.2)$ & 9.66 & +46.2 & +0.04 & -0.07 & -0.174 & 2008-12-02 & \\
\hline 053721 & 0.193 & 2.69 & 1.75 & 9.89 & +59.5 & -0.25 & +0.14 & -0.010 & 2008-12-02 & planet-host star \\
\hline 054375 & 0.353 & 4.06 & 2.44 & 9.36 & +35.9 & -0.12 & -0.08 & +0.147 & $2008-05-26$ & \\
\hline 055459 & 0.218 & 2.39 & 1.58 & 9.58 & +51.1 & -0.04 & +0.03 & +0.072 & 2007-02-05 & \\
\hline 055868 & 0.256 & 1.92 & 2.14 & 9.55 & -26.9 & -0.02 & -0.01 & -0.166 & 2007-04-19 & \\
\hline 059589 & 0.205 & 1.81 & $(<1.2)$ & 9.39 & -110.2 & +0.05 & -0.24 & -0.020 & 2008-05-21 & \\
\hline 059610 & 0.229 & 2.22 & 1.62 & 9.63 & +59.1 & -0.13 & +0.07 & -0.066 & 2007-02-06 & planet-host star \\
\hline 062175 & 0.198 & 2.52 & 1.83 & 9.53 & -78.2 & -0.27 & -0.14 & +0.150 & 2008-05-21 & \\
\hline 062816 & 0.296 & 3.57 & 2.16 & 9.49 & +31.8 & -0.03 & -0.08 & +0.069 & 2007-02-07 & \\
\hline 063048 & 0.189 & 1.81 & $(<1.1)$ & 9.50 & -107.6 & -0.20 & -0.02 & -0.020 & $2008-05-25$ & \\
\hline 063636 & 0.351 & 2.93 & 2.26 & 9.62 & +22.0 & -0.01 & +0.18 & -0.027 & 2007-04-19 & \\
\hline 064150 & 0.187 & 2.21 & $(<1.0)$ & 9.63 & -14.4 & +0.01 & +0.03 & +0.067 & 2007-02-05 & \\
\hline 064747 & 0.204 & 1.77 & $(<1.1)$ & 9.80 & -70.1 & -0.08 & -0.01 & -0.193 & $2008-05-21$ & \\
\hline 070319 & 0.207 & 1.56 & 1.09 & 10.07 & -103.0 & -0.06 & -0.03 & -0.341 & 2007-04-19 & \\
\hline 072604 & 0.191 & 2.26 & $(<1.1)$ & 10.03 & -122.9 & -0.23 & -0.19 & -0.147 & $2008-05-22$ & \\
\hline 075676 & 0.200 & 2.18 & $(<1.1)$ & 9.62 & -6.0 & -0.03 & -0.08 & -0.090 & $2008-08-14$ & \\
\hline 076114 & 0.195 & 1.76 & 0.91 & 9.59 & -34.0 & +0.06 & +0.00 & -0.003 & $2008-08-14$ & \\
\hline 077749 & 0.416 & 4.77 & 2.70 & 9.22 & +79.9 & +0.15 & +0.12 & +0.234 & $2008-05-27$ & \\
\hline 078217 & 0.213 & 1.17 & 1.89 & 9.54 & -10.8 & -0.02 & +0.09 & -0.215 & $2008-05-22$ & \\
\hline 079672 & 0.193 & 2.34 & 1.63 & 9.66 & +1.7 & -0.06 & -0.03 & +0.039 & 2007-02-06 & \\
\hline 085042 & 0.187 & 2.01 & $(<0.8)$ & 9.43 & -95.8 & +0.00 & -0.02 & +0.035 & 2008-08-14 & \\
\hline 085810 & 0.193 & 2.86 & 2.05 & 9.43 & +88.7 & -0.07 & +0.10 & +0.154 & 2008-08-14 & \\
\hline 088194 & 0.196 & 1.78 & $(<0.8)$ & 9.67 & -49.2 & -0.04 & +0.01 & -0.071 & $2008-08-15$ & \\
\hline 088945 & 0.558 & 6.51 & $(<1.1)$ & 9.73 & +11.4 & -0.08 & +0.42 & -0.025 & 2008-08-14 & outlier $A_{\mathrm{Li}}$ (Fig. 5) \\
\hline 089282 & 0.240 & 3.41 & 2.45 & 9.34 & +77.5 & -0.21 & +0.02 & +0.001 & $2008-05-21$ & \\
\hline 089474 & 0.189 & 2.50 & $(<0.9)$ & 9.89 & -6.1 & -0.25 & +0.04 & +0.010 & 2008-08-14 & \\
\hline 089912 & 0.436 & 6.04 & 2.69 & 9.43 & +62.9 & -0.12 & +0.24 & +0.036 & $2008-05-26$ & \\
\hline 090004 & 0.176 & 1.76 & $(<1.2)$ & 9.83 & -157.4 & -0.01 & -0.14 & -0.013 & $2008-05-22$ & planet-host star \\
\hline 091287 & 0.185 & 2.05 & 1.74 & 9.21 & -111.6 & +0.01 & -0.11 & -0.004 & $2008-05-21$ & \\
\hline 096184 & 0.165 & 2.03 & 1.65 & 9.25 & +64.8 & -0.08 & +0.01 & +0.107 & $2008-05-26$ & \\
\hline 096395 & 0.218 & 2.30 & 2.18 & 9.76 & +10.9 & -0.05 & -0.02 & -0.119 & 2008-08-14 & \\
\hline 096402 & 0.182 & 1.99 & $(<1.1)$ & 9.90 & -115.2 & -0.17 & -0.01 & -0.040 & $2008-05-25$ & \\
\hline 096901 & 0.185 & 1.97 & $(<1.1)$ & 9.77 & -24.2 & -0.12 & +0.00 & +0.086 & $2008-05-27$ & planet-host star \\
\hline 096948 & 0.175 & 1.85 & 1.52 & 9.62 & -8.8 & +0.00 & +0.06 & +0.097 & $2008-05-26$ & \\
\hline 097420 & 0.209 & 2.57 & 2.22 & 9.65 & +32.3 & +0.00 & +0.04 & +0.064 & 2008-05-21 & \\
\hline 098921 & 0.329 & 3.66 & 2.47 & 9.13 & +25.7 & -0.01 & +0.17 & +0.158 & $2008-05-27$ & \\
\hline 100963 & 0.200 & 2.39 & 1.72 & 9.71 & -1.6 & -0.03 & -0.02 & -0.012 & $2008-05-22$ & \\
\hline 104075 & 0.409 & 5.01 & 2.72 & 9.20 & +85.6 & -0.12 & +0.09 & +0.033 & $2008-05-27$ & \\
\hline 109110 & 0.409 & 4.78 & 2.49 & 9.28 & +63.3 & +0.04 & +0.19 & +0.052 & 2008-08-12 & \\
\hline 110205 & 0.216 & 1.98 & 1.09 & 9.96 & -46.0 & -0.12 & +0.06 & -0.215 & 2008-08-11 & \\
\hline 112504 & 0.209 & 1.99 & 2.01 & 9.63 & -16.0 & -0.06 & -0.01 & +0.020 & 2008-08-11 & \\
\hline 113579 & 0.573 & 10.31 & 3.06 & 9.57 & -1.8 & -0.24 & +0.48 & +0.049 & 2008-08-11 & \\
\hline 113989 & 0.222 & 1.94 & $(<0.8)$ & 10.13 & -274.9 & -0.13 & -0.20 & -0.482 & 2008-08-13 & \\
\hline 115715 & 0.215 & 2.34 & 1.72 & 10.08 & -85.6 & -0.29 & +0.06 & -0.201 & 2008-08-12 & \\
\hline 116613 & 0.349 & 2.96 & 2.12 & 8.95 & +60.1 & -0.05 & +0.12 & +0.127 & $2008-08-12$ & \\
\hline Sun/Moon & 0.193 & 2.29 & 0.92 & 9.66 & $\cdots$ & $\ldots$ & $\ldots$ & $\cdots$ & 2007-02-07 & \\
\hline
\end{tabular}

Notes. Following the HIP number (Col. 1), $r_{0}(8542)$ is the residual flux at the line center of the Ca II 8542 line (Col. 2), $v_{\mathrm{e}} \sin i$ is the projected rotational velocity in $\mathrm{km} \mathrm{s}^{-1}$ calibrated in this study (cf. Sect. 3) (Col. 3), $A_{\mathrm{Li}}$ is the non-LTE lithium abundance (derived in Sect. 4.3 of Paper I and expressed in the usual normalization of $\left.A_{\mathrm{H}}=12.00\right)(\mathrm{Col} .4)$, and $\log$ age is the logarithm of the stellar age (in yr) evaluated from the evolutionary tracks (log $a g e_{\text {trk }}$ defined in Sect. 3.3 of Paper I) (Col. 5). In Cols. 6-9 are presented the star-Sun differences of $T_{\text {eff }}$ (effective temperature), log $g$ (surface gravity), $v_{\mathrm{t}}$ (microturbulence), and $\mathrm{Fe} / \mathrm{H}$ (metallicity), respectively, which were established precisely in Paper I by using the complete differential analysis (cf. Sect. 3.1.2 therein). Column 10 gives the observational date of each OAO spectrum, which we used for the measurement of $r_{0}(8542)$. 